\title{
System Model of Underground UWB Based on MB-OFDM
}

\author{
Yanjing Sun ${ }^{1,2}$, Beibei Zhang ${ }^{1}$ \\ ${ }^{1}$ School of Information \& Electrical Engineering, China University of Mining and Technology, \\ Xuzhou, China \\ ${ }^{2}$ State Key Laboratory of Coal Resources and Safe Mining, China University of Mining and Technology, \\ Xuzhou, China \\ E-mail:Yanjingsun_cn@163.com,zhangbeibei@163.com \\ Received October 11, 2010; revised November 8, 2010; accepted November 10, 2010
}

\begin{abstract}
To overcome the frequency-selective fading of ultra wide band (UWB) caused by the multi-path propagation in complicated environment of coal mines, an underground wireless communication system of UWB based on multi-band orthogonal frequency division multiplexing (MB-OFDM) for coal mine is presented. Independent angle impulse response is used to build up revised channel model for underground tunnels based on the traditional S-V model. With the revised channel model, we construct the underground UWB system based on MB-OFDM and give the band division scheme and the correction approach for channel. The model of the system was simulated in the complex environment of coal mine. Simulation results and analysis show that the underground wireless communication system of UWB based on MB-OFDM can effectively withstand multipath fading and has the advantages of high anti-noise ability, low bit-error rate, and wide coverage.
\end{abstract}

Keywords: Channel Model, Tunnel, Underground UWB, OFDM

\section{Introduction}

In key areas of safety monitoring in coal mine such as the tunnel, working face, tunneling surface and minedout area, wireless communication system can overcome the problem of deployment and maintenance for wired information transmission system. And it can realize wireless detection of coal mining, tunneling face, gob gas, and reconstruct the environment monitoring system after the disaster quickly. It has great significance to improve the remnant of coal mine safety monitoring system under the condition of disaster $[1,2]$. But, the complex natural environment and operating conditions in coal mine restrict the development of mine wireless communication seriously. Because of Ultra-Wide band (UWB) Wireless Communication with a high transfer rate, low power consumption, anti-interference, it is conducive to resolve the limited issue of radio communication under the complex environment in coal mines [3]. For research on wireless communications in coal mine, Sun [4] studied the wireless signal propagation characteristics under the mine environment. Moe [5] analyzed of the application of UWB in the tunnel, and in theory, and described the feasibility of the UWB system used in the coal mine tunnels. Peng [6] researched the UWB signals' propagation characteristics in the coal mine tunnels from the perspective of channel path loss.

In order to effectively overcome the path loss, multipath delay and different channels power attenuation of mine roadway, it need to model a UWB system that can be a true reflection of propagation characteristics of UWB signal under the multi-path environment. In this paper, with the characteristics of multi-band orthogonal frequency division multiplexing [7-9] against multipath delay, based on the modified channel loss model, we establish the MB-OFDM based UWB communication system model of coal mine. The simulation results show that the system can guarantee the high communication speed and strong anti-jamming capabilities under mine complex environment.

\section{Channel Model}

In the process of wireless communication underground coal mine, the received signals are stacked by the reflection, diffraction and scattering of multi-path signals. The radio frequencies of communication in this environment 
need not to be licensed. We use the S-V model of recommended cluster-based IEEE802.15.3a as a basis to improve the channel model $[5,6]$. Based on time-frequency interleaving (TFI) of the MB-OFDM-UWB as the sub-band group, in which center frequencies are respectively $3432 \mathrm{MHz}, 3960 \mathrm{Mhz}$ and $4488 \mathrm{MHz}[7,8]$. Taking into account that the multi-path channel is a typical frequency selective channel, the different frequency bands and bandwidth have different frequency response characteristics and temporal resolution. So it is necessary to transform the channel. After transformation, the channel embodies the characteristics of different sub-band at the same time to adapt to different information rates. Here, an example of 3.168-3.696 GHz sub-band, according to frequency components, different frequency bands use the appropriate sampling rate, to achieve not only satisfy the sampling theorem also minimize the amount of data. And in order to satisfy the requirement of the data system' transfer clock $\left(T_{\mathrm{P}}\right)$, it need interpolation and extraction for channel.

Let frequency characteristic as $h_{1}(t)$. The multi-path resolution is $0.167 \mathrm{~ns}$. In order to turn $h_{1}(t)$ into $h^{\prime}(t)$ whose resolution is $T_{\mathrm{P}}$. That is, the sample rate is turned into $T_{\mathrm{P}} / T$ times. First, the signal is deal with $\mathrm{N}$-fold interpolation and $M$-fold extraction after low-pass filter. Finally, $T_{\mathrm{P}} / T=N / M$. The concrete process is shown as Figure 1.

\section{System Model}

\subsection{Improved Channel Model}

In $\mathrm{S}-\mathrm{V}$ model, the impulse response of channel is expressed as (1) [7]:

$$
h(t)=X \sum_{n=1}^{N} \sum_{k=1}^{K(n)} \alpha_{n k} \delta\left(t-T_{n}-\tau_{n k}\right)
$$

$X$ is a lognormal distributed random variable representing the magnitude of channel gain. $N$ is the observed number of clusters. $K(n)$ is the received number of multipath in the $n^{\text {th }}$ cluster. $\alpha_{n k}$ is coefficients of the $k^{\text {th }}$ path in the nth cluster. $T_{n}$ is the arrival time of the $n$th cluster. $\tau^{n k}$ is the $k^{\text {th }}$ path delay in the $n$th cluster.

In the time domain, multipath propagation channel is shown clustered distribution pattern, and is closely linked with the multi-path component arrival time and arrival angle. Therefore, channel model should be related with the time and azimuth, its impulse response

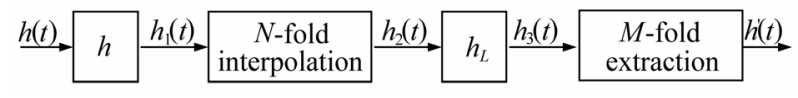

Figure 1. Process of channel transformation. expression is:

$$
h(t, \theta)=X \sum_{n=1}^{N} \sum_{k=1}^{K(n)} \alpha_{n k} e^{j \theta_{n k}} \delta\left(t-T_{n}-\tau_{n k}\right)
$$

If there is correlation between arrival time and arrival angle of the multi-path component, then the larger the cluster mean-square displacement angle, the longer delay, and [4] has studied that arrival time and arrival angle are not related about channel characteristic. It believed that they were both independent. So think it $h(t, \theta) \approx h(t) h(\theta)$, to the $h(\theta)$ completely independent of, so:

$$
h(\theta)=\sum_{n=1}^{N} \sum_{k=1}^{K(n)} \delta\left(\theta-\omega_{n k}\right)
$$

$\omega_{n k}$ is the $n$th path arrival angle in the $n$th cluster. $h(\theta)$ is angle impulse response similar to time impulse response of channel.

Table 1 shows the environmental characteristics of tunnel channels and the required parameters of channel by simulation. We take paths within $10 \mathrm{~dB}$ of peak power or 85 percent the total power as the main path. In order to determine the values of parameters, we firstly need to obtain statistic parameters of these characteristics. After that, we use the fitting method to get the concrete parameters. Because of the coal mine tunnel has different environmental characteristics with the variety indoors channels, the parameters after fitting can reflect the corresponding characteristics.

Using the parameters in table, we run simulation under the channel environment. The impulse response we get is shown as Figure 2.

In the figure, we can obviously observe multipath clusters which are verified with the actual measurement of UWB multipath clusters. This is a typical result of multi-path fading channel. Then we can see exponential attenuation of received power. In a short time, the power attenuation tends to zero after $300 \mathrm{~ns}$. It achieves the effect of reducing the power consumption. Meanwhile, the delay spread characteristics showed that it has more than 40 ns delay multipath component for the UWB in mine tunnel. It will introduce the very serious inter-symbol interference in underground UWB communication systems.

\subsection{MB-OFDM}

MB-OFDM is a special multi-carrier modulation (MCM) method. It includes a wide frequency band which is divided into a few sub-bands. Using MB- OFDM modulation and demodulation method with the larger number of sub-carriers, the amplitude spectrum of signals superposed by one of each sub-carrier has good 
Table 1. Environmental characteristics and parameters of channel model.

\begin{tabular}{ccccc}
\hline $\begin{array}{c}\text { Channel } \\
\text { number. }\end{array}$ & $\begin{array}{c}\text { Channels } \\
\text { characteristic }\end{array}$ & $\Lambda(1 / \mathrm{ns})$ & $\lambda(1 / \mathrm{ns})$ & $\Gamma$ \\
\hline 500 & Extreme & 0.0667 & 2.1 & 36 \\
$\gamma$ & $\sigma \xi(\mathrm{dB})$ & $\sigma \zeta(\mathrm{dB})$ & $\sigma g(\mathrm{~dB})$ & Type \\
24 & 3.3941 & 3.3941 & 3 & LOS \\
\hline
\end{tabular}

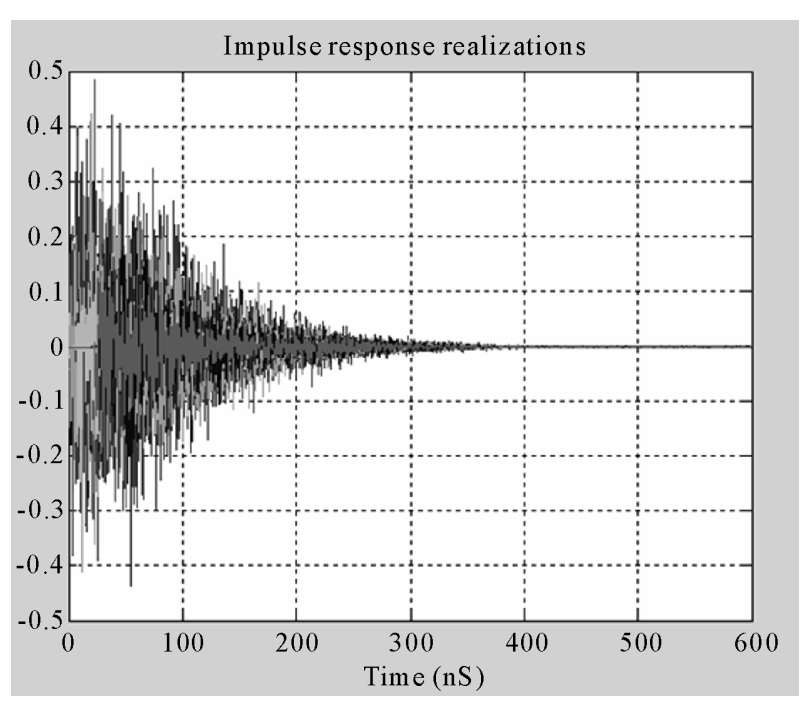

Figure 2. Impulse response of discrete channel.

matrix characteristics. So it can make UWB system to achieve high spectral efficiency. According to interference between various UWB devices or between UWB devices and other wireless communication devices, as well as various different sources of interference in mine tunnels, it can decrease one or more sub-band transmission power, or cancel the launch of one or more sub-bands, to effectively reduce the interference. It can also inhibit the corresponding sub-car- riers and more precisely control the spectrum shape of synthesis signals.

\subsection{Simulation Model}

Figure 3 is the simulation model of mine UWB communication systems based on MB-OFDM in Simulink. The generation method of synthesis OFDM signals is similar to the traditional OFDM system. But the specific parameters such as symbol length, sub-carrier spacing, and cyclic prefix length have greater difference with traditional systems. The signal is send out by the antenna. Through the revised UWB channel, they arrive at the receivers. The received signal are processed through the inverse mapping, deinterleave, descrambling and others. It will output the corresponding data finally.

\section{Performance Simulation and Analysis}

\subsection{Performance}

Beside the fading and the interferences, the received symbol is affected by AWGN whose variance is $N_{0}$, as reference of the average SNR per QPSK symbol can be defined as:

$$
\overline{\gamma_{\delta}}(\varepsilon, \tau)=\frac{E_{S} \sigma_{H}^{2}}{\delta_{C}^{2}+\delta_{S}^{2}+N_{0}}
$$

Since the energy per bit $E_{b}=E_{\delta} / 2$, the average SNR per bit is:

$$
\overline{\gamma_{b}}(\varepsilon, \tau)=(1 / 2) \overline{\gamma_{\delta}}(\varepsilon, \tau)
$$

Moreover, we can define $S N R_{\gamma}$, which is the SNR at the receiver as follow:

$$
S N R_{\gamma}=E_{b} / N_{0}
$$

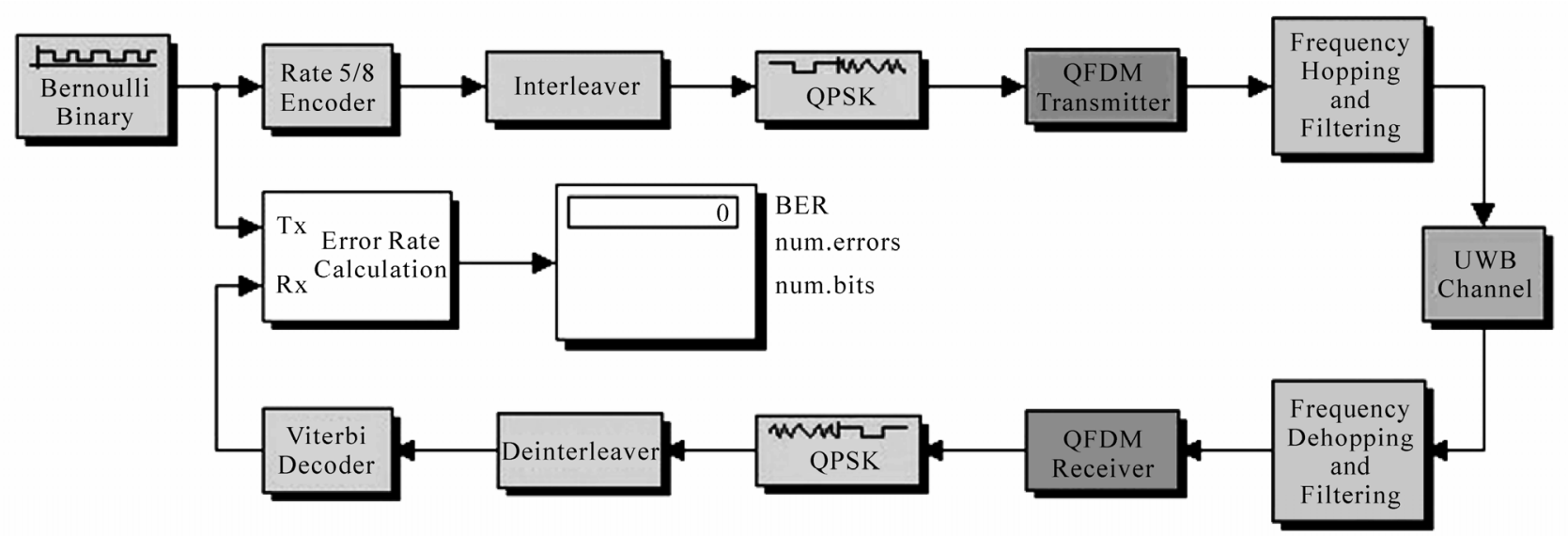

Figure 3. Simulink simulation of OFDM-UWB in coal mine. 
where the $E_{b}$ is the average received energy per bit, and $N_{0}$ is the single-sided power spectral density of the additive white Gaussian noise, i.e., the noise variance.

Average bit error rate (BER) is defined as a ratio of the bits error received to the whole of bits sent. BER is measures the unconditional performance for the channel model of OFDM. As [10], using the distribution of $|H[k]|^{2}$, we can get the average BER of our OFDM UWB scheme also. Average BER in the channel model can be classified two classes such as average uncoding $U C_{B E R}$ (Uncoded BER) and $C_{B E R}$ (Coded BER). Their symbols can be defined as follow:

$$
U C_{B E R}=E\left[Q\left(\sqrt{2|h|^{2} S N R_{\gamma}}\right)\right]=\frac{1}{2}\left(1-\sqrt{\frac{S N R_{\gamma}}{1+S N R_{\gamma}}}\right)
$$

where the $Q$ is the complementary cumulative distribution function of the standard nor-mal distribution.

After the convolutional decoder, the average coded BER is defined. Each coded bit has an equal probability of being wrongly decoded. Convolutional encoder is followed by an ideal time-frequency interleaves.

$$
C_{B E R} \leq\left.\frac{\partial T(U, I)}{\partial I}\right|_{I=1, U=\sqrt{4 U C_{B E R}\left(1-C_{B E R}\right)}}
$$

where $T(U, I)$ is the generating function of the convolutional encoder. We define $d_{\text {free }}$ as the minimum free distance of convolutional code, and define $N_{b}$ as the sum of Hamming weight of all the input sequences. From the previous derivation, the $C_{B E R}$ can be defined as follow:

$$
C_{B E R} \approx N_{b}\left[4 U C_{B E R}\left(1-U C_{B E R}\right)\right]^{\frac{d_{\text {free }}}{2}}
$$

And, therefore, we arrive at:

$$
C_{B E R} \approx N_{b}\left[1+S N R_{\gamma}\right]^{-\frac{d_{\text {free }}}{2}}
$$

\subsection{Simulation Analysis}

According to the mine's physical environment, we may improve bandwidth utilization by increasing the useful data sub-carriers, improving code rate, or reducing the cyclic prefix, guard interval length and training sequence length. In the following simulation, the number of OFDM symbols in each frame is 60 . Simulation includes anti-noise capacity, bit error rate and coverage areas of the UWB system based on MB-OFDM in coal mine.

1) Anti-noise capability

In accordance with the characteristics of multi-path frequency selective fading in coal mine, signal is emitted from the OFDM transmitter. The dynamic range of OFDM signal is about $30 \mathrm{~dB}$. The transmitter emitted signals in different frequency bands. When $\mathrm{SNR}=60 \mathrm{~dB}$, Figure 4 shows the received signal constellation hash map with the availability and unavailability of channel estimation. As shown in the mine environment, the signal amplitude and phase shift have been skewing seriously when the SNR $=60 \mathrm{~dB}$ and the unavailability of channel estimation circumstances. However, with channel estimation and synchronization, the phase has been fully corrected and the receiver signal distribution was evenly distributed X-shaped.

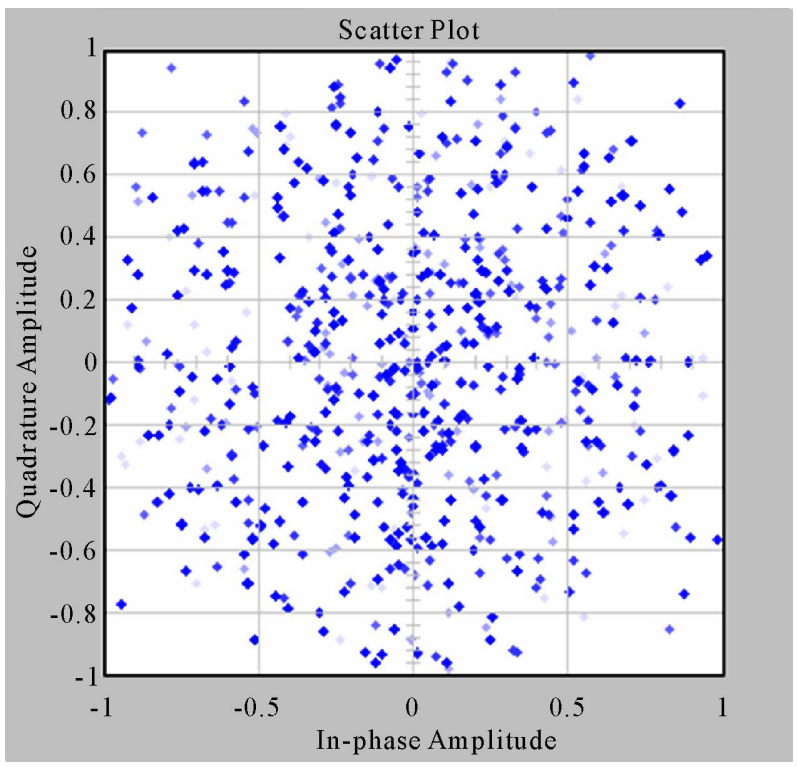

(a)

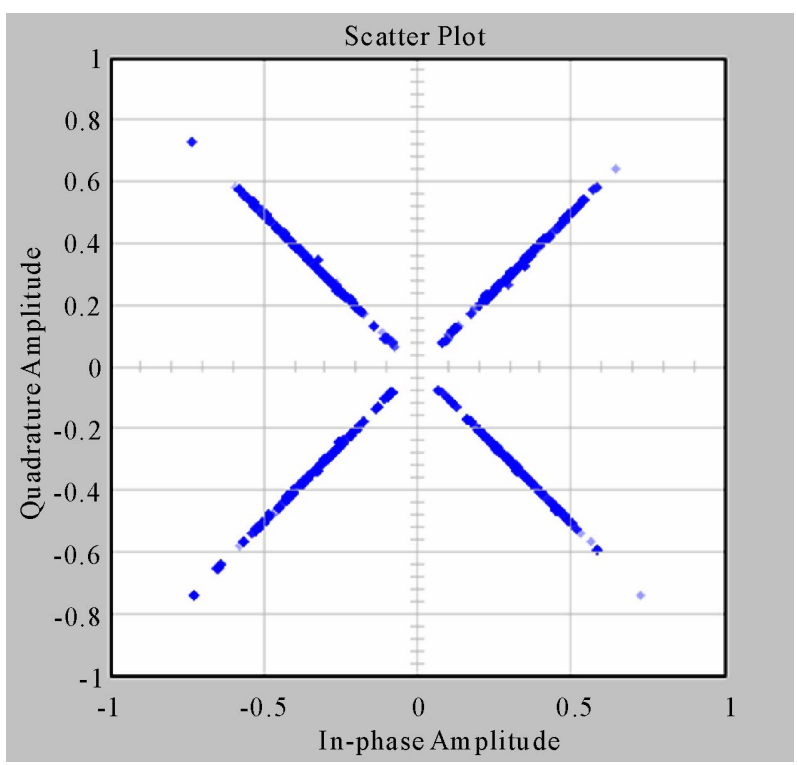

(b)

Figure 4. Constellation of received signal when SNR $=60 \mathrm{~dB}$. (a) No channel estimation; (b) Channel estimation and synchronization. 


\section{2) BER and SNR}

Figure 5 is the curve of bit error rate (BER) with signal noise ratio (SNR). OFDM works in the QPSK modulation for each value. In the OFDM-UWB wireless systems, sub-band bandwidth is $528 \mathrm{MHz}$ and SNR range is $8 \mathrm{~dB}-17 \mathrm{~dB}$.

3) Coverage range

When system data transfer speed is $110 \mathrm{Mbps}$ and $480 \mathrm{Mbps}$ respectively and the possibility of channel connectivity is not less than $90 \%$, the relationship between the transmission distance and packet error rate (PER) is shown in Figure 6.

As shown, simulation results are basically consistent with the theoretical value. The little difference is because of the distribution of $h(t)$.

\section{Conclusions}

In this paper, we combine the characteristics of the resistance against multi-path delay of multi-band OFDM and

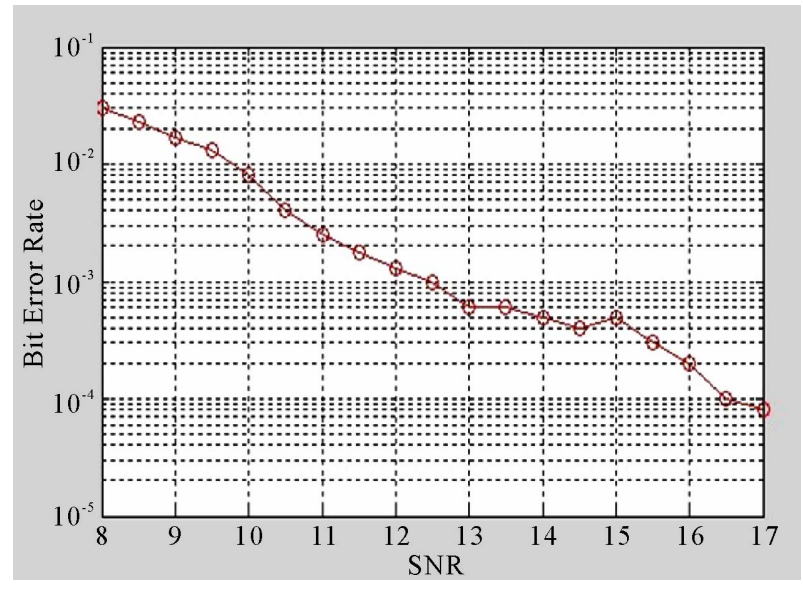

Figure 5. BER along with SNR.

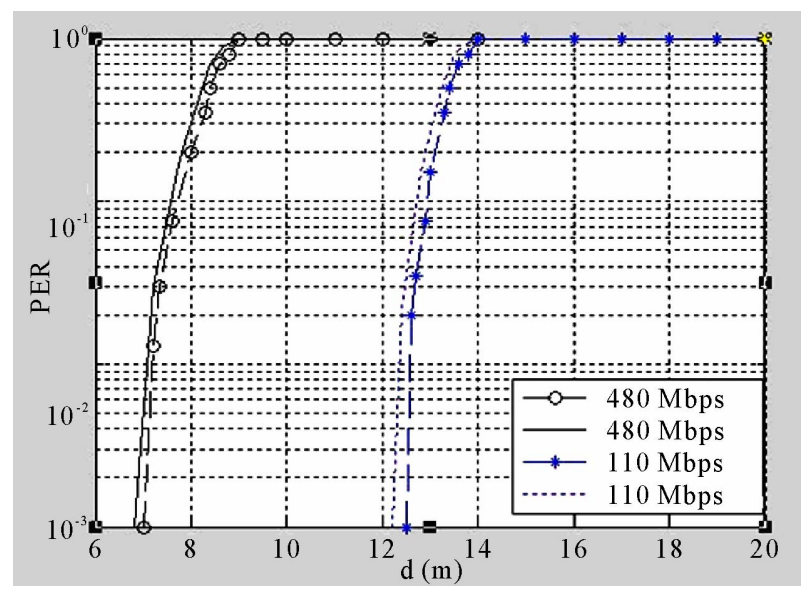

Figure 6. PER at $110 \mathrm{Mbps}$ and $480 \mathrm{Mbps}$ (... and - are simulation values respectively). the correction of channel loss model. A mode of wireless communication system based MB-OFDM-UWB has been established. The simulation shows that the system can be effective against the complex multi-path fading underground mine environment, with high anti-noise ability, low bit error rate and wide coverage.

\section{Acknowledgements}

This work is partly supported by National Science Foundation of China under grant No. 50904070, the China Postdoctoral Science Foundation No. 20100471009, the Key Project of the National High Technology Research and Development Program of China (863 Program) under grant No. 2008AA062200, the National High Technology Research and Development Program of China (863 Program) under grant No. 2007AA01Z180, and the Science foundation of China University of mining and technology under Grant No. OC080303.

\section{References}

[1] C. Baouche, A. Freitas and M. Misson, "Radio Proximity Detection in a WSN to Localize Mobile Entities within a Confined Area," Journal of Communications, Vol. 4, No. 4, 2009, pp. 232-240.

[2] Y. Wu, M. Lin and I. J. Wassell, "Modified 2D Finite-Difference Time-Domain Based Tunnel Path Loss Prediction for Wireless Sensor Network Applications," Journal of Communications, Vol. 4, No. 4, 2009, pp. 214 223.

[3] W. Yang, X. S. Feng and J. P. Sun, "The Theories and Key Technologies for the New Generation Mine Wireless Information System," Journal of China Coal Society, Vol. 29, No. 4, 2004, pp. 506-611.

[4] J. P. Sun and Q. D. Shi, "Study on Electric Magnetic Transmission in Mine Roadway," Coal Science and Technology, Vol. 29, No. 1, 2001, pp. 25-27.

[5] Z. W. Moe, C. George and R. S. Nelson, "Performance of Rake Reception in Dense Multipath Channels: Implications of Spreading Band-Width and Selection Diversity Order," IEEE Journal on Selected Areas in Communications, Vol. 18, No. 8, 2000, pp. 1516-1525. doi:10.1109/ 49.864015

[6] P. Li, Y. J. Sun, J. S. Qian, et al., "Wireless Channel Model of UWB for Coal Mine Tunnels," Journal of Huazhong University of Science and Technology, Vol. 36, No. s1, 2008, pp. 582-583.

[7] Y.-F. Wang, H.-Z. Yu, S. Zhang, G.-G. Bi, et al., “Analysis of the Characteristic of Monocycle Waveform in Coal Mine UWB Communication System," Journal of China University of Mining and Technology, Vol. 35, No. 2, 2006, pp. 167-169.

[8] C. Q. Li, "Some Key Techniques Researches on the UWB-OFDM Systems," Beijing University of Post and 
Telecommunication, Beijing, 2006.

[9] W.-J. Dong, N.-H. Yu and Y. Yin, "A Novel Time-Frequency Coded Multi-Rate MB-OFDM UWB System Model," Journal of Electronics \& Information Technology, Vol. 28, No. 10, 2006, pp. 1858-1861.
[10] Q. Y. Zou, A. Tarighat and A. H. Sayed, "Performance Analysis of Multi-band OFDM UWB Communications with Application to Range Improvement," IEEE Transactions on Vehicular Technology, Vol. 56, No. 6, 2007, pp. 3864-3878. doi:10.1109/TVT.2007.901957 